\title{
Minimization of maximum lateness in an $m$-machine permutation flow shop with a general exponential learning effect
}

\begin{abstract}
In this paper, we consider the permutation flow shop scheduling problem with a general exponential learning effect. The objective is to minimize the maximum lateness. A special case that can be solved to optimality by EDD algorithm is provided. To form a hybrid solution framework, several heuristics, a branch-and-bound algorithm and a new Nested-partition-based solution approach are proposed. Composite bounds and dominance rules are developed to reduce the searching region and to provide guidance on the lower bound. Finally, computational experiments are conducted to evaluate the performance of the algorithms.
\end{abstract}

Keywords: Scheduling; Flow shop; Learning effect; Branch and bound; Heuristic algorithm; Nested Partition (NP);

\section{Introduction}

For many years, scheduling problems have been widely studied and applied to different industrial environment of production. With the booming development of information technique, research on scheduling problem gains new impetus. As for classical research on scheduling problems as well as commercial scheduling systems, the processing time of a job is usually assumed to be constant. In many modern manufacturing environment where human power and cyclic production play a very significant role, however, both machines and workers can improve their performance and shorten the processing time by repeating similar production operations. Thus, the phenomenon known as learning effect which indicts that the processing time of a job is likely to be shorter when it is sequenced later can be found in many realistic situations. For example, in the assembly workshop where the processing time is highly dependent on a worker's skill, knowledge and the organization ability, the repetition would teach workers the more efficient way of producing (Kuo and Yang 2006). Therefore, with the enhancement of production skills and knowledge, the worker will be able to conduct the assembly operation much faster. In terms of the scheduling system, the consideration of the learning effect in some manufacturing environments can be helpful in improving the accuracy of the planning and productivity estimation. The neglect of the effect of this phenomenon on the scheduling problem, on the other hand, would lead to the deviation from the actual value of the processing time and the underestimation of the production capacity. Since Wright (1936) first brought the idea of learning effect in the manufacturing 
environment, there have been many industrial cases supporting the theory(Day 1983; Venezia 1985; Webb 1994). As for the introduction of the learning theory into the scheduling problem, Biskup (1999) and Cheng and Wang (2000) were among the pioneers. Readers can refer to Biskup (2008) and Janiak and Rudek (2009) for extensive surveys on scheduling problems with learning effect.

In many discrete manufacturing facilities each job has to undergo a series of operations (Pinedo 2002). As one of the most common manufacturing environment and a conventional research topic, the flow shop scheduling problem has received considerable attention and has been widely discussed. It has been shown that, except for some certain cases (Johnson 1954), polynomial solutions can be hardly found for most of the flow shop problems. (Gonzalez and Sahni 1978). Many approximation algorithms have been developed to cope with the NP-hard problem (Garey and Johnson 1979; Smutnicki 1998, etc.). For research on the objective function of minimizing the maximum lateness in scheduling environment including single-machine, flow shop, etc., readers can refer to Haouari and Ladhari (2000), Allahverdi and Aldowaisan (2004), Bachman and Janiak (2000) and Hsua and Lin (2000).

As for flow shop scheduling with a learning effect, it has drawn a growing attention recently. A two-machine flow shop scheduling problem with the position-based learning effects is considered in Lee and $\mathrm{Wu}$ (2004). For minimizing the total completion time, both heuristic algorithms and a branch-and-bound algorithm are developed. Cheng et al. (2004) provide a comprehensive survey on scheduling with time-dependent processing times. In addition, scheduling problems with processing times that are highly dependent on the start time are discussed. Wang and Xia (2005) consider a flow shop scheduling problem with the learning effect to minimize the makespan and the total flow time. For some certain instances, they find the polynomial time solutions. To extend the work, Xu, et al. (2008) provide the worst-case bounds for SPT algorithm. The objective considered, on the other hand, includes minimizing the total weighted completion time, the discounted total weighted completion time and the sum of the quadratic job completion times. Koulamas and Kyparisis (2007) study the two-machine flow shop models with both position-based and sum-of-job-processing-times-based learning effects. The optimality of SPT algorithm for certain cases is shown. Cheng et al. (2008) introduce a new model where the processing time is dependent on the sum of the processing time of the jobs already processed as well as the job's position. Both the single machine and the flow shop scheduling problem are discussed. In Cheng et al. (2013), scheduling with a position-weighted learning effect based on sum-of-logarithm-processing-times and job position is considered. Worst-case bounds and several analytical theorems are provided for both single-machine and flow shop scheduling problems. As for the general situation, the heuristic algorithms and the corresponding worst-case analysis are conducted. Both a single-machine and an $m$-machine flow shop scheduling models with the sum-of-processing-time-based learning effect are studied in Wu and Lee (2009). Cases 
that could be solved in polynomial time are provided. As for the manufacturing environment of a flow shop with the general-position-based learning effect, i.e., the actual processing time of a job is defined by a general non-increasing function of its scheduled position, Wang and Wang (2011) propose several heuristic algorithms and the corresponding worst-case bounds for different regular objective functions. Kuo et al.(2012) study a flow shop scheduling problem with the sum-of-processing-times-based learning effect. The heuristic algorithms and the associated worst-case analysis are also furnished. Several regular objective functions are considered in the paper. In Wang et al. (2013), a truncation parameter as well as the learning ratio is incorporated into the learning effect. Worst-case bounds are provided for different regular objective functions in the condition of busy schedules. Flow shop scheduling problems with the exponential learning effects are considered in Wang $(2012,2014)$. In Wang and Wang (2012), the processing time is position-based, whereas it is sum-of-processing-time-based in Wang and Wang (2014). Heuristics for several objective functions and their corresponding worst-case analysis are developed in both of the research.

In Biskup (2008), the learning models are classified into two classes: position-based learning and sum-of-processing-time-based learning. It could be more appropriate to make the assumption that position-based learning effects take place in the case of processing independent operations like machine setup. Sum-of-processing-time-based learning effects, on the other hand, would be a better assumption if human iterations have great impact on the job processing. The realistic implication of the sum-of-processing-time-based learning effect is that the more time a worker has devoted to a skill practising, the better performance he can achieve. The practical scenario of the former class is the highly standardized manufacturing like chips/circuit board manufacturing, the running of bottling plants (Cheng et al. 2013) and cellphone assembly workshop. The latter class, on the other hand, could be exemplified by aircraft maintenance (Cheng et al. 2013) and producing high-end machinery like satellites and petroleum drills. In this paper, we consider the flow shop scheduling problem with a general exponential learning effect combining both of the classes of the learning effect(Bai et al. (2012)). Both of the position and the sum of the processing time are considered in the learning ratio. The objective of minimization of the maximum lateness is first introduced into the discussion of the model. The model is appropriate when the learning effects of both independent operations and human iterations exist. The practical example is the surface defects inspection for the steel plates or bars. It this case, since there are many kinds of plates/bars and each plate/bar may have its own defect characteristic, it requires both of the setup operation and the defect inspection. In addition, for each plate/bar, the area/length can significantly influence the learning effect. As a result, both of the job position and the sum of the processing times should be considered. The other example is the cutting operation in the paper industry. Since there are many sizes of the final products, both of the setup of the cutting machine and the area of the raw material can 
affect the learning effect. Hence, the learning ratio should include the job position as well as the sum of the processing times. Similar cases can be seen in the operations of drilling holes in parts and rubber mixing in the tyre manufacturing industry. Our main contribution includes the analysis of the mathematical properties of the problem, the proposition of a branch-and-bound algorithm with a composite bound, several heuristics and a solution framework based on Nested Partition method and mathematical bounds techniques, namely Bounds-based-nested-partition (BBNP).

The remainder of the paper is organized as follows. Notations, assumptions and the analysis of the scheduling models are given in Section 2. Section 3 provides a special case that can be solved to optimality by EDD. In Section 4 and Section 5, several dominance rules and a composite bound for the problem studied are developed. The specific procedure of the branchand-bound, heuristic algorithms and the solution framework are proposed in Section 6. Detailed experimental results are displayed in Section 7. Finally, the summary of the paper is presented in Section 8.

\section{Notations and assumptions}

The model considered here is based on a general flow shop scheduling environment where there are $m$ machines in series and each job of $n$ in the system follows the same route and has to be processed on each of the machines. As the environment of permutation flow shop defines, the permutation of the job on each machine remains the same, which means First In First Out principle is followed and each operation will be operated right after the pre-operation of its own job and the operation sequenced earlier on the same machine are finished. Specifically, the flow shop scheduling problem consists of scheduling $n$ jobs $J=\left\{J_{1}, J_{2}, \ldots, J_{n}\right\}$ on $m$ machines $M_{1}, M_{2}, \ldots, M_{m}$. Each job $J_{j}$ consists of a chain operations $\left(O_{1 j}, O_{2 j}, \ldots, O_{m j}\right)$ and must be processed without preemption on each machine with permutation schedule. The (normal) processing time of operation $O_{i j}$ is denoted by $p_{i j}$. The actual processing time $p_{i j r}$ of job $J_{j}$ on machine $M_{i}$ is a function dependent on both its position $r$ and the sum of the processing time of the operations positioned earlier on the same machine in a schedule. In this paper, we consider flow shop scheduling with a general exponential time-dependent learning effect:

$$
p_{i j r}=p_{i j}\left(\alpha a^{\sum_{l=1}^{r-1} p_{i[l]}}+\beta\right) b^{r-1}, i=1,2, \ldots, m ; r, j=1,2, \ldots, n,
$$

where $\alpha \geq 0, \beta \geq 0,0<a, b \leq 1$ is the learning rate, and $\alpha+\beta=1$. For a given schedule $\pi$, let $C_{i j}(\pi)=C_{i j}$ be the completion time of operation $O_{i j}, i=1,2, \ldots, m ; j=1,2, \ldots, n$, $C_{j}(\pi)=C_{j}=C_{m j}$ represent the completion time of job $J_{j}, L_{j}$ be the lateness of a job $j$, i.e. $L_{j}=C_{j}-d_{j}$ where $d_{j}$ is the due time for job $j$. The objective is to obtain a schedule to minimize the maximum lateness, i.e., $L_{\max }=\max \left\{L_{j} \mid j=1,2, \ldots, n\right\}$. When $\alpha=0, \beta=$ $1, a>0$ and $b=1$, the problem is reduced to a standard permutation flow shop problem with 
the objective of minimizing maximum lateness. It is well known that the problem $F m \mid p_{i j r}=$ $p_{i j}\left(\alpha a^{\sum_{l=1}^{r-1} p_{i[l]}}+\beta\right) b^{r-1} \mid L_{\max }(m \geq 3)$ is NP-complete.(Johnson 1954)

For the better presentation in the following, we denote $\sigma, \sigma_{1}$ and $\sigma_{2}$ as partial sequence of jobs on top of the global sequence; $\sigma^{\prime}, \sigma_{1}^{\prime}$ and $\sigma_{2}^{\prime}$ as the complementary sequence of $\sigma, \sigma_{1}$ and $\sigma_{2}$ in $\pi$.

To dig further into the problem, it can be equally transformed into another form by introducing tail-operations for each job. The two equivalent forms provide two different views of the problem. Specifically, we denote $d_{\max }=\max _{j} d_{j}$. Assume the tail operations are processed on an imaginary machine without any capacity constraint, i.e. they might be operated in parallel for all jobs. Then for each job $j$, a tail operation with the processing time $t_{j}\left(t_{j}=d_{\max }-d_{j}\right)$ is added into the scheduling system. We denote $C_{j}^{\text {tail }}$ as the completion time of each job $j$ after finishing the tail-operation; $C_{\text {max }}^{\text {tail }}$ as the makespan after the tail-operation of the last job, i.e. $C_{\max }^{\text {tail }}=\max \left\{C_{j}^{\text {tail }} \mid j=1,2, \ldots, n\right\}$. A tail operation begins right after the last operation of the job is finished, which implies $C_{j}^{t a i l}=C_{m j}+t_{j}$. Consequently, a new flow shop model $F m\left|p_{i j r}=p_{i j}\left(\alpha a^{\sum_{l=1}^{r-1} p_{i[l]}}+\beta\right) b^{r-1}, t_{j}\right| C_{\max }^{\text {tail }}$ is introduced and the following lemma can be obtained.

Lemma 1 The problem $F m\left|p_{i j r}=p_{i j}\left(\alpha a^{\sum_{l=1}^{r-1} p_{i[l]}}+\beta\right) b^{r-1}\right| L_{\max }$ is equivalent to $F m \mid p_{i j r}=$ $p_{i j}\left(\alpha a^{\sum_{l=1}^{r-1} p_{i[l]}}+\beta\right) b^{r-1}, t_{j} \mid C_{\max }^{\text {tail }}$.

Proof By definition, $L_{\max }=\max _{j}\left(C_{m j}-d_{j}\right)$. Since it stands in the new model that $t_{j}=d_{\max }-d_{j}$, we could obtain that $L_{\max }=\max _{j} C_{j}^{\text {tail }}-d_{\max }$. Thus, $L_{\max }=C_{\max }^{\text {tail }}-d_{\max }$.

\section{Special cases}

In the following, we offer a special case that can be solved to optimality in polynomial time.

Lemma 2 (Bai et al. (2009)) For the problems $1\left|p_{j r}=p_{j}\left(\alpha a^{\sum_{l=1}^{r-1} p_{[l]}}+\beta\right) b^{r-1}\right| C_{\max }$, an optimal schedule can be obtained by sequencing the jobs in non-decreasing order of $p_{j}$ (i.e., the SPT rule).

Theorem 1 For the problem $F m\left|p_{i j r}=p_{j}\left(\alpha a^{\sum_{l=1}^{r-1} p_{i[l]}}+\beta\right) b^{r-1}\right| L_{\max }$, if jobs have the same processing time on each machine and agreeable weights, i.e., $p_{j} \leq p_{k}$ implies $d_{j} \leq d_{k}$ for all jobs $J_{j}$ and $J_{k}$, an optimal schedule can be obtained by sequencing the jobs in non-decreasing order of $d_{j}$ (EDD rule).

Proof Suppose $\pi$ is a schedule not arranged as EDD rule defines, which means that there are at least two adjacent job positions, say $j$ and $(j+1)$, such that $d_{j}>d_{j+1}$ and $p_{[j]} \geq p_{j+1}$. Without loss of generality and for reasons of simplicity, in the following formulas we assume 
that the jobs are ordered in sequence $1,2,3,, j-1$ before the $j_{t h}$ job. Denote $\sigma$ as the sequence including the jobs that are positioned before $j$ and still arranged in an EDD way. Schedule $\tilde{\pi}$ is obtained from schedule $\pi$ by interchanging jobs in the $j$ th and in the $(j+1)$ th positions of $\pi$. Correspondingly, under $\pi$,

$$
\begin{gathered}
L_{[j]}(\sigma j(j+1))=p_{1}+\ldots+p_{j-1}\left(\alpha a^{\sum_{l=1}^{j-2} p_{l}}+\beta\right) b^{j-2}+p_{j}\left(\alpha a^{\sum_{l=1}^{j-1} p_{l}}+\beta\right) b^{j-1}+ \\
(m-1) \max \left\{p_{1}, \ldots, p_{j-1}\left(\alpha a^{\sum_{l=1}^{j-2} p_{l}}+\beta\right) b^{j-2}, p_{j}\left(\alpha a^{\sum_{l=1}^{j-1} p_{l}}+\beta\right) b^{j-1}\right\}-d_{j} \\
L_{[j+1]}(\sigma j(j+1))=p_{1}+\ldots+p_{j-1}\left(\alpha a^{\sum_{l=1}^{j-2} p_{l}}+\beta\right) b^{j-2}+p_{j}\left(\alpha a^{\sum_{l=1}^{j-1} p_{l}}+\beta\right) b^{j-1}+p_{j+1}\left(\alpha a^{\sum_{l=1}^{j-1} p_{l}+p_{j}}+\beta\right) b^{j}+ \\
(m-1) \max \left\{p_{1}, \ldots, p_{j-1}\left(\alpha a^{\sum_{l=1}^{j-2} p_{l}}+\beta\right) b^{j-2}, p_{j}\left(\alpha a^{\sum_{l=1}^{j-1} p_{l}}+\beta\right) b^{j-1}, p_{j+1}\left(\alpha a^{\sum_{l=1}^{j-1} p_{l}+p_{j}}+\beta\right) b^{j}\right\}-d_{j+1} \\
L_{[j]}(\sigma(j+1) j)=p_{1}+\ldots+p_{j-1}\left(\alpha a^{\sum_{l=1}^{j-2} p_{l}}+\beta\right) b^{j-2}+p_{j+1}\left(\alpha a^{\sum_{l=1}^{j-1} p_{l}}+\beta\right) b^{j-1}+ \\
(m-1) \max \left\{p_{1}, \ldots, p_{i-1}\left(\alpha a^{\sum_{l=1}^{j-2} p_{l}}+\beta\right) b^{j-2}, p_{j+1}\left(\alpha a^{\sum_{l=1}^{j-1} p_{l}}+\beta\right) b^{j-1}\right\}-d_{j+1} \\
L_{[j+1]}(\sigma(j+1) j)=p_{1}+\ldots+p_{j-1}\left(\alpha a^{\sum_{l=1}^{j-2} p_{l}}+\beta\right) b^{j-2}+p_{j+1}\left(\alpha a^{\sum_{l=1}^{j-1} p_{l}}+\beta\right) b^{j-1}+p_{j}\left(\alpha a^{\sum_{l=1}^{j-1} p_{l}+p_{j+1}}+\beta\right) b^{j}+ \\
(m-1) \max \left\{p_{1}, \ldots, p_{j-1}\left(\alpha a^{\sum_{l=1}^{j-2} p_{l}}+\beta\right) b^{j-2}, p_{j+1}\left(\alpha a^{\sum_{l=1}^{j-1} p_{l}}+\beta\right) b^{j-1}, p_{j}\left(\alpha a^{\sum_{l=1}^{j-1} p_{l}+p_{j+1}}+\beta\right) b^{j}\right\}-d_{j}
\end{gathered}
$$

Since $d_{j} \geq d_{j+1}, p_{j} \geq p_{j+1}$, it is easy to show that $\max \left\{L_{[j]}(\sigma j(j+1)), L_{[j+1]}(\sigma j(j+1))\right\}=$ $L_{[j+1]}(\sigma j(j+1))$ and $L_{[j+1]}(\sigma j(j+1)) \geq L_{[j]}(\sigma(j+1) j)$.

According to Lemma 2, we can obtain that

$$
\begin{array}{r}
p_{1}+\ldots+p_{j-1}\left(\alpha a^{\sum_{l=1}^{j-2} p_{l}}+\beta\right) b^{j-2}+p_{j}\left(\alpha a^{\sum_{l=1}^{j-1} p_{l}}+\beta\right) b^{j-1}+p_{j+1}\left(\alpha a^{\sum_{l=1}^{j-1} p_{l}+p_{j}}+\beta\right) b^{j} \\
\geq p_{1}+\ldots+p_{j-1}\left(\alpha a^{\sum_{l=1}^{j-2} p_{l}}+\beta\right) b^{j-2}+p_{j+1}\left(\alpha a^{\sum_{l=1}^{j-1} p_{l}}+\beta\right) b^{j-1}+p_{j}\left(\alpha a^{\sum_{l=1}^{j-1} p_{l}+p_{j+1}}+\beta\right) b^{j}
\end{array}
$$

In addition,

$$
\begin{array}{r}
(m-1) \max \left\{p_{1}, \ldots, p_{j-1}\left(\alpha a^{\sum_{l=1}^{j-2} p_{l}}+\beta\right) b^{j-2}, p_{j}\left(\alpha a^{\sum_{l=1}^{j-1} p_{l}}+\beta\right) b^{j-1}, p_{j+1}\left(\alpha a^{\sum_{l=1}^{j-1} p_{l}+p_{j}}+\beta\right) b^{j}\right\} \\
\geq(m-1) \max \left\{p_{1}, \ldots, p_{j-1}\left(\alpha a^{\sum_{l=1}^{j-2} p_{l}}+\beta\right) b^{j-2}, p_{j+1}\left(\alpha a^{\sum_{l=1}^{j-1} p_{l}}+\beta\right) b^{j-1}, p_{j}\left(\alpha a^{\sum_{l=1}^{j-1} p_{l}+p_{j+1}}+\beta\right) b^{j}\right\}
\end{array}
$$

Thus,

$$
\max \left\{L_{[j]}(\sigma(j+1) j), L_{[j+1]}(\sigma(j+1) j)\right\} \leq \max \left\{L_{[j]}(\sigma j(j+1)), L_{[j+1]}(\sigma j(j+1))\right\}
$$




\section{Dominance properties}

Dominance properties can be helpful in trimming the redundant nodes and hence improving the efficiency when applying the branch-and-bound algorithm. In the following, two dominance properties are presented to cope with the problem.

Lemma 3 In terms of flow shop scheduling, for the partial sequences $\sigma_{1}$ and $\sigma_{2}$ of the same jobs, if $C\left(\sigma_{1}\right) \leq C\left(\sigma_{2}\right)$ and $L\left(\sigma_{1}\right) \leq L\left(\sigma_{2}\right)$, the subtree rooted at the schedule $\sigma_{2}$ can be eliminated.

Proof Since $C\left(\sigma_{1}\right) \leq C\left(\sigma_{2}\right)$, it is easy to show that $L\left(\sigma_{1}^{\prime}\right) \leq L\left(\sigma_{2}^{\prime}\right)$. With the other condition $L\left(\sigma_{1}\right) \leq L\left(\sigma_{2}\right)$, it stands that $L\left(\sigma_{1} \sigma_{1}^{\prime}\right) \leq L\left(\sigma_{2} \sigma_{2}^{\prime}\right)$.

Lemma 4 In terms of flow shop scheduling, given partial sequence $\sigma$ and unscheduled jobs $i$ and $j$, if $C(\sigma i j)-d_{j} \leq L(\sigma j)$, then the subtree rooted at the sequence $\sigma j i$ can be eliminated and the sequence oij could be further digged into.

Proof It is easy to show that $L_{i}(\sigma i j) \leq L_{i}(\sigma j i)$. In addition, $C(\sigma i j)-d_{j} \leq L(\sigma j)$, which means that $\max \left(L_{i}(\sigma j i), L(\sigma j)\right) \geq L(\sigma i j)$.

\section{Lower bounds}

The lower bounds for the partial solutions have great impact on the efficiency of a branch-andbound algorithm. In the following, a composite bound for partial solutions is presented. The computation is implemented from three different perspectives with the help of Lemma 2 and Lemma 5. For a better presentation, we denote each bound from a certain perspective as $L B(1)$, $L B(2)$ or $L B(3)$. Let $(\pi=P S, U S)$ be a schedule of jobs in which $P S$ is the sequence of the scheduled jobs, and assume there are $k$ jobs in $P S$, and $U S$ is any sequence of the unscheduled jobs with $n-k$ jobs. The derivation of the lower bound is based on the completion time of the job on machine $M_{i}(1 \leq i \leq m)$.

Lemma 5 In terms of flow shop scheduling, given partial sequence $\sigma$, the lower bound of the maximum lateness of any schedule could be presented as $L B=L B\left(C_{\max }\left(\sigma \sigma^{\prime}\right)\right)-\max _{j \in(\pi \backslash \sigma)} d_{j}$.

Proof By definition, it is clear that $\max _{j \in \pi} L_{j}\left(\sigma \sigma^{\prime}\right) \geq \min \left(C_{\max }\left(\sigma \sigma^{\prime}\right)-d_{j^{*}}\right)$, where $d_{j^{*}}$ is the due time of the job that is positioned in the end of the sequence. In addition,

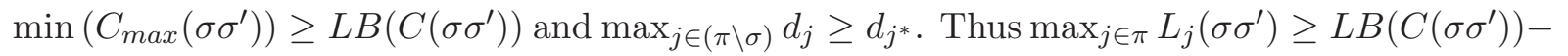
$\max _{j \in(\pi \backslash \sigma)} d_{j}$.

Except for the above proof, Lemma 5 can also be derived from lemma 1 according to the relationship that $L_{\max }=C_{\max }^{t a i l}-d_{\max }$. 


\subsection{Lower bound 1}

As the definition goes, the completion time of the $(k+1)$ th job on machine $M_{i}$ is

$$
\begin{aligned}
C_{i[k+1]}(\pi) & =\max \left\{C_{i-1,[k+1]}(\pi), C_{i[k]}(\pi)\right\}+p_{i[k+1]}\left(\alpha a^{\sum_{l=1}^{k} p_{i[l]}}+\beta\right) b^{k} \\
& \geq C_{i[k]}(\pi)+p_{i[k+1]}\left(\alpha a^{\sum_{l=1}^{k} p_{i[l]}}+\beta\right) b^{k} .
\end{aligned}
$$

In a similar way,

$$
\begin{gathered}
C_{m[k+1]}(\pi) \geq C_{i[k]}(\pi)+\sum_{u=i}^{m} p_{u[k+1]}\left(\alpha a^{\sum_{l=1}^{k} p_{u[l]}}+\beta\right) b^{k}, \\
C_{m[k+j]}(\pi) \geq C_{i[k]}(\pi)+\sum_{u=i}^{m-1} p_{u[k+1]}\left(\alpha a^{\sum_{l=1}^{k} p_{u[l]}}+\beta\right) b^{k}+\sum_{h=1}^{j} p_{m[k+h]}\left(\alpha a^{\sum_{l=1}^{k} p_{m[l]}+\sum_{l=1}^{h-1} p_{m[k+l]}}+\beta\right) b^{k+h-1},
\end{gathered}
$$

where $1 \leq j \leq n-k$.

Therefore, the makespan can be shown as

$C_{\max }=C_{[n]}(\pi) \geq C_{i[k]}(\pi)+\sum_{u=i}^{m-1} p_{u[k+1]}\left(\alpha a^{\sum_{l=1}^{k} p_{u[l]}}+\beta\right) b^{k}+\sum_{h=1}^{n-k} p_{m[k+h]}\left(\alpha a^{\sum_{l=1}^{k} p_{m[l]}+\sum_{l=1}^{h-1} p_{m[k+l]}}+\beta\right) b^{k+h-1}$.

Note that the term $C_{i[k]}(\pi)$ is a fixed constant, and a lower bound for $P S$ can be obtained by minimizing the $\sum_{h=1}^{n-k} p_{m[k+h]}\left(\alpha a^{\sum_{l=1}^{k} p_{m[l]}+\sum_{l=1}^{h-1} p_{m[k+l]}}+\beta\right) b^{k+h-1}$ and $\sum_{u=i}^{m-1} p_{u[k+1]}\left(\alpha a^{\sum_{l=1}^{k} p_{u[l]}}+\beta\right) b^{k}$. From Lemma 2 , the term $\sum_{h=1}^{n-k} p_{m[k+h]}\left(\alpha a^{\sum_{l=1}^{k} p_{m[l]}+\sum_{l=1}^{h-1} p_{m[k+l]}}+\beta\right) b^{k+h-1}$ is minimized by arranging the jobs in non-decreasing order of $p_{m[j]}$. Consequently, combining Lemma 5 , the lower bound for machine $M_{i}$ is

$$
\begin{gathered}
L B_{i}=C_{i[k]}(\pi)+\sum_{u=i}^{m-1} p_{u[k+1]^{*}}\left(\alpha a^{\sum_{l=1}^{k} p_{u[l]}}+\beta\right) b^{k} \\
+\sum_{h=1}^{n-k} p_{m(k+h)}\left(\alpha a^{\sum_{l=1}^{k} p_{m[l]}+\sum_{l=1}^{h-1} p_{m(k+l)}}+\beta\right) b^{k+h-1}-\max _{j \in k+1, k+2, \ldots, n}\left\{d_{j}\right\},
\end{gathered}
$$

where $[k+1]^{*}$ refers to the first unfinished job $\mathrm{k}+1$ that would generate the minimal addition of the processing time on machines from $i$ to $m-1, p_{m(k+1)} \leq p_{m(k+2)} \leq \ldots \leq p_{m(n)}$ is a nondecreasing order of the normal processing times on $M_{m}$ for the remaining unscheduled jobs.

To tighten the lower bound, the maximum value $L B(1)_{i}, i=1,2, \ldots, m$ is chosen as a lower bound for PS, i.e.,

$$
L B(1)=\max \left\{L B(1)_{1}, L B(1)_{2}, \ldots, L B(1)_{m}\right\} .
$$




\subsection{Lower bound 2}

As for Lower bound 2, in terms of the makespan of the schedule, the similar perspective that each lower bound is obtained from focusing on a certain machine is adopted, except that all the remaining operations in each machine are considered instead of the only one operation costing least time and adjacent to the latest finished one. As the definition stands, the completion time of the $(k+1)$ th job on machine $M_{i}$ is

$$
C_{i[k+1]}(\pi) \geq C_{i[k]}(\pi)+p_{i[k+1]}\left(\alpha a^{\sum_{l=1}^{k} p_{i[l]}}+\beta\right) b^{k} .
$$

In a similar way,

$$
\begin{gathered}
C_{m[k+1]}(\pi) \geq C_{i[k]}(\pi)+\sum_{u=i}^{m} p_{u[k+1]}\left(\alpha a^{\sum_{l=1}^{k} p_{u[l]}}+\beta\right) b^{k}, \\
C_{m[k+j]}(\pi) \geq C_{i[k]}(\pi)+\sum_{h=1}^{j} p_{i[k+h]}\left(\alpha a^{\sum_{l=1}^{k} p_{i[l]}+\sum_{l=1}^{h-1} p_{i[k+l]}}+\beta\right) b^{k+h-1} \\
+\sum_{u=i+1}^{m} p_{u[k+j]}\left(\alpha a^{\sum_{l=1}^{k} p_{u[l]}+\sum_{l=1}^{j-1} p_{u[k+l]}}+\beta\right) b^{k+j-1},
\end{gathered}
$$

where $1 \leq j \leq n-k$.

Therefore, the makespan is

$C_{\max }=C_{[n]}(\pi) \geq C_{i[k]}(\pi)+\sum_{h=1}^{n-k} p_{i[k+h]}\left(\alpha a^{\sum_{l=1}^{k} p_{i[l]}+\sum_{l=1}^{h-1} p_{i[k+l]}}+\beta\right) b^{k+h-1}+\sum_{u=i+1}^{m} p_{u[n]}\left(\alpha a^{\sum_{l=1}^{n-1} p_{u[l]}}+\beta\right) b^{n-1}$.

Note that the term $C_{i[k]}(\pi)$ is a fixed constant, and a lower bound for $P S$ can be obtained by minimizing the $\sum_{h=1}^{n-k} p_{i[k+h]}\left(\alpha a^{\sum_{l=1}^{k} p_{i[l]}+\sum_{l=1}^{h-1} p_{i[k+l]}}+\beta\right) b^{k+h-1}$ and $\sum_{u=i+1}^{m} p_{u[n]}\left(\alpha a^{\sum_{l=1}^{n-1} p_{u[l]}}+\beta\right) b^{n-1}$.

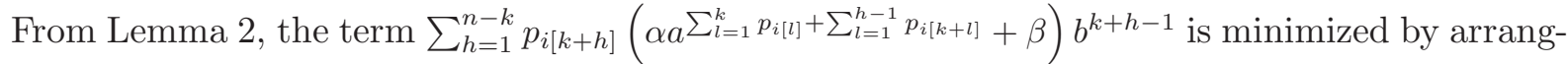
ing the jobs in non-decreasing order of $p_{i[j]}$. Consequently, combining Lemma 5 , the lower bound for machine $M_{i}$ is

$$
\begin{aligned}
L B_{i} & =C_{i[k]}(\pi)+\sum_{h=1}^{n-k} p_{i(k+h)}\left(\alpha a^{\sum_{l=1}^{k} p_{i[l]}+\sum_{l=1}^{h-1} p_{i(k+l)}}+\beta\right) b^{k+h-1} \\
& +\sum_{u=i+1}^{m} p_{u[n]^{*}}\left(\alpha a^{\sum_{l=1}^{n-1} p_{u[l]}}+\beta\right) b^{n-1}-\max _{j \in k+1, k+2, \ldots, n}\left\{d_{j}\right\},
\end{aligned}
$$

where $[n]^{*}$ refers to the last unfinished job $n$ that would generate the minimal addition of the processing time on machines from $i+1$ to $m, p_{i(k+1)} \leq p_{i(k+2)} \leq \ldots \leq p_{i(n)}$ is a nondecreasing order of the normal processing times on $M_{i}$ for the remaining unscheduled jobs.

To tighten the lower bound, the maximum value $L B(2)_{i}, i=1,2, \ldots, m$ is chosen as a lower bound for PS, i.e.,

$$
L B(2)=\max \left\{L B(2)_{1}, L B(2)_{2}, \ldots, L B(2)_{m}\right\} .
$$




\subsection{Lower bound 3}

In terms of Lower bound 3, the job-based-perspective is taken into the computation. The lower bound for the completion time on machine $m$ is calculated according to the arbitrary position of a certain job. As the definition stands, the completion time of the $(k+1)$ th job on machine $M_{i}$ is

$$
\begin{aligned}
C_{i[k+1]}(\pi) & =\max \left\{C_{i-1,[k+1]}(\pi), C_{i[k]}(\pi)\right\}+p_{i[k+1]}\left(\alpha a^{\sum_{l=1}^{k} p_{i[l]}}+\beta\right) b^{k} \\
& \geq C_{1[k]}(\pi)+\sum_{u=1}^{i} p_{u[k+1]}\left(\alpha a^{\sum_{l=1}^{k} p_{u[l]}}+\beta\right) b^{k} .
\end{aligned}
$$

In a similar way,

$$
\begin{aligned}
& C_{m[k+1]}(\pi) \geq C_{1[k]}(\pi)+\sum_{u=1}^{m} p_{u[k+1]}\left(\alpha a^{\sum_{l=1}^{k} p_{u[l]}}+\beta\right) b^{k}, \\
& C_{i[k+j]}(\pi) \geq C_{1[k]}(\pi)+\sum_{h=1}^{j-(w+1)} p_{i[k+w+h+1]}\left(\alpha a^{\sum_{l=1}^{k} p_{i[l]}+\sum_{l=1}^{w+1} p_{i[k+l]}+\sum_{l=1}^{h-1} p_{i[k+w+l+1]}}+\beta\right) b^{k+w+h} \\
& +\sum_{h=1}^{w} p_{1[k+h]}\left(\alpha a^{\sum_{l=1}^{k} p_{1[l]}+\sum_{l=1}^{h-1} p_{1[k+l]}}+\beta\right) b^{k+h-1}+\sum_{u=1}^{i} p_{u[k+w+1]}\left(\alpha a^{\sum_{l=1}^{k} p_{u[l]}+\sum_{l=1}^{w} p_{u[k+l]}}+\beta\right) b^{k+w}
\end{aligned}
$$

where $1 \leq j \leq n-k, 1 \leq w \leq j-k$.

$$
\begin{aligned}
& C_{m[k+j]}(\pi) \geq C_{1[k]}(\pi)+\sum_{h=1}^{j-(w+1)} p_{m[k+w+h+1]}\left(\alpha a^{\sum_{l=1}^{k} p_{m[l]}+\sum_{l=1}^{w+1} p_{m[k+l]}+\sum_{l=1}^{h-1} p_{m[k+w+l+1]}}+\beta\right) b^{k+w+h} \\
& +\sum_{h=1}^{w} p_{1[k+h]}\left(\alpha a^{\sum_{l=1}^{k} p_{1[l]}+\sum_{l=1}^{h-1} p_{1[k+l]}}+\beta\right) b^{k+h-1}+\sum_{u=1}^{m} p_{u[k+w+1]}\left(\alpha a^{\sum_{l=1}^{k} p_{u[l]}+\sum_{l=1}^{w} p_{u[k+l]}}+\beta\right) b^{k+w},
\end{aligned}
$$

where $1 \leq j \leq n-k, 1 \leq w \leq j-k$.

Therefore, the makespan is

$$
\begin{aligned}
& C_{\max }=C_{[n]}(\pi) \geq C_{1[k]}(\pi)+\sum_{h=1}^{n-(k+w+1)} p_{m[k+w+h+1]}\left(\alpha a^{\sum_{l=1}^{k} p_{m[l]}+\sum_{l=1}^{w+1} p_{m[k+l]}+\sum_{l=1}^{h-1} p_{m[k+w+l+1]}}+\beta\right) b^{k+w+h} \\
& +\sum_{h=1}^{w} p_{1[k+h]}\left(\alpha a^{\sum_{l=1}^{k} p_{1[l]}+\sum_{l=1}^{h-1} p_{1[k+l]}}+\beta\right) b^{k+h-1}+\sum_{u=1}^{m} p_{u[k+w+1]}\left(\alpha a^{\sum_{l=1}^{k} p_{u[l]}+\sum_{l=1}^{w} p_{u[k+l]}}+\beta\right) b^{k+w} .
\end{aligned}
$$

where $1 \leq w \leq n-k$.

Note that the term $C_{1[k]}(\pi)$ is a fixed constant, and a lower bound for $P S$ can be obtained by minimizing the $\sum_{h=1}^{n-(k+w+1)} p_{m[k+w+h+1]}\left(\alpha a^{\sum_{l=1}^{k} p_{m[l]}+\sum_{l=1}^{w+1} p_{m[k+l]}+\sum_{l=1}^{h-1} p_{m[k+w+l+1]}}+\beta\right) b^{k+w+h}$ 


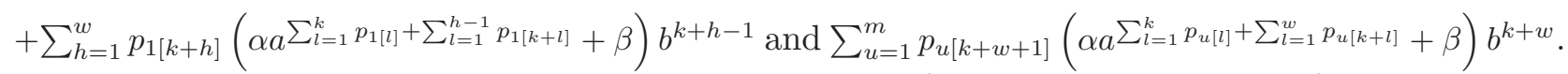

In addition, according to Lemma 2 , the term $\sum_{h=1}^{w} p_{1[k+h]}\left(\alpha a^{\sum_{l=1}^{k} p_{1[l]}+\sum_{l=1}^{h-1} p_{1[k+l]}}+\beta\right) b^{k+h-1}$

$+\sum_{h=1}^{n-(k+w+1)} p_{m[k+w+h+1]}\left(\alpha a^{\left.\sum_{l=1}^{k} p_{m[l]}+\sum_{l=1}^{w+1} p_{m[k+l]}+\sum_{l=1}^{h-1} p_{m[k+w+l+1]}+\beta\right) b^{k+w+h}}\right.$ is minimized by sequencing the jobs other than the $(k+w+1)_{t h}$ job in non-decreasing order of $\min \left\{p_{1[j]}, p_{m[j]}\right\}$. As for the part that reflects the accumulated learning effect, it is minimized when the process time of the past position is taken as $\max \left\{p_{1[j]}, p_{m[j]}\right\}$. Consequently, combining Lemma 5 , the lower bound for job $J_{j}$ is

$$
\begin{gathered}
L B_{j}=C_{1[k]}(\pi)+\sum_{u=1}^{m} p_{u j}\left(\alpha a^{\sum_{l=1}^{k} p_{u[l]}+\sum_{l=1}^{n-k-1} p_{u[k+l]}}+\beta\right) b^{n-1} \\
+\sum_{h=1}^{n-k-1} p_{\min (k+h)}\left(\alpha a^{\left.\sum_{l=1}^{k} p_{1[l]}+\sum_{l=1}^{h-1} p_{\max (k+h)}+\beta\right) b^{k+h-1}-\max _{j \in k+1, k+2, \ldots, n}\left\{d_{j}\right\},}\right.
\end{gathered}
$$

where $k \leq j \leq n-k, k \leq h \leq n-k, p_{u j}$ is the processing time of job $J_{j}$ on machine $M_{u}, p_{\min (k+h)}$ refers to the processing time of the queue in the non-decreasing order of $\min \left\{p_{1[k+h]}, p_{m[k+h]}\right\}$, while $p_{\max (k+h)}$ is the maximal processing time of each operation in the above queue. Hence, $p_{\min (k+1)} \leq p_{\min (k+2)} \leq \ldots \leq p_{\min (n)}$ is a nondecreasing order of the normal processing times for the remaining unscheduled jobs.

To tighten the lower bound, the maximum value $L B(3)_{j}, j=1,2, \ldots, n-k$ is chosen as a lower bound for PS, i.e.,

$$
L B(3)=\max \left\{L B(3)_{1}, L B(3)_{2}, \ldots, L B(3)_{n-k}\right\} .
$$

Given the above lower bounds from three different perspectives, the final form of the composite lower bound can be expressed as bellows.

$$
L B=\max \{L B(1), L B(2), L B(3)\} .
$$

\section{Algorithms}

\subsection{Two heuristic algorithms}

Due to the NP-complete property of the problem, in the following, several local-search-based heuristics will be proposed to provide a fast way of solving as well as the upperbound for the branch-and-bound algorithm. Specifically in this paper, with the proposition of Lemma 1 and Lemma 2, a modified version of $O\left(n^{2}\right)$ heuristic we denote as BJ of Bachman and Janiak (2000) and $O\left(m n^{4}\right)$ FL heuristic of Framinan and Leisten (2003) are introduced as fast methods of solving problem $F m\left|p_{i j r}=p_{i j}\left(\alpha a^{\sum_{l=1}^{r-1} p_{i[l]}}+\beta\right) b^{r-1}\right| L_{\max }(m \geq 3)$. Bellow shows the specific procedure of those algorithms. 


\section{BJ algorithm}

Step 1. Arrange the jobs in ascending order of $\sum_{i=1}^{m} p_{i j}, i=1,2,3, j=1,2, \ldots, n$. (SPT rule of flow shop scheduling)

Step 2. Find the position of the job $j_{\max }$ with the maximum job lateness in the schedule obtained.

Step 3. In the subset of the jobs before $j_{\max }$, i.e., $j<j_{\max }$, find the position of the job $j_{m}$ with the larger due time than job $j_{\max }$, i.e., $d_{j_{m}}>d_{j_{\max }}$. The searching is sequentially conducted from job $j_{\max }$ to job 0. If no job is found, STOP; otherwise, go to Step 4.

Step 4. Move the job $j_{s}$ to the position that is right after the job $j_{\max }$ and calculate the objective function again. If the objective value is enhanced, go to Step 2; otherwise, go to Step 3 and update the beginning searching point as $j_{m}$.

\section{FL algorithm}

Step 1. Arrange the jobs in ascending order of $d_{j}$. (EDD rule)

Step 3: Set $k=2$. Select the first two jobs from the sorted list and select the better between the two possible sequences. Note the objective function compared here is the makespan

of problem of $F m\left|p_{i j r}=p_{i j}\left(\alpha a^{\sum_{l=1}^{r-1} p_{i[l]}}+\beta\right) b^{r-1}, t_{j}\right| C_{\max }$, the objective value of which is denoted as $C_{\text {max }}^{\text {tail }}$.

Step 4: Increment $k, k=k+1$. Select the $k$ th job from the sorted list and insert it into $k$ possible positions of the best partial sequence obtained so far. Among the $k$ sequences, the best $k$-job partial sequence is selected based on minimum $C_{m a x}^{t a i l}$. Next, determine all possible sequences by interchanging jobs in positions $i$ and $j$ of the above partial sequence for all $i, j$ $(1 \leq i<k, i<j \leq k)$. Select the best partial sequence among $k(k-1) / 2$ sequences having minimum $C_{\text {max }}^{\text {tail }}$.

Step 5: If $k=n$, then STOP and output the result of $C_{m a x}^{\text {tail }}-d_{m a x}$; otherwise, go to Step 4 .

\subsection{Branch-and-bound algorithm}

The branch-and-bound algorithm assigns jobs in a forward manner starting from the first position. In the searching tree, we choose a branch and systematically work down the tree until we either eliminate it on grounds of a lower bound or reach its final node, which is either used as a substitute for the initial solution or eliminated. Our branch-and-bound algorithm is given as follows:

Step 1. The BJ and FL algorithms are applied to obtain an initial solution (choosing a better solution). 
Step 2. A depth-first search is used in the branching procedure until all the nodes are explored or eliminated. Start the assignment of jobs at the beginning of a schedule and move forward one step at a time.

Step 3. In the $k$ th level node, the first $k$ positions are occupied by $k$ specific jobs. Select one of the remaining $n-k$ jobs for the node at level $k+1$.

Step 4. Calculate the lower bound for $C_{\max }$ of the unfathomed partial schedules or $C_{\max }$ of the completed schedules. During the search, any unfathomed branch that has a lower bound which is larger than or equal to the initial solution is fathomed, i.e., eliminated from further consideration. If the value of the completed schedule is less than the initial solution, use it as the new initial solution. Otherwise, eliminate it.

Step 5. Continue to search all the nodes, and the remaining initial solution is optimal.

\subsection{Bounds-based-nested-partition approach}

In this subsection, we propose a new solution method combining Nested Partition optimization framework, local-search-based heuristics and lower bounds technique. We denote the method as Bounds-based Nested Partition (BBNP). It is based on Nested Partition optimization framework (NP), a newly proposed random solution framework dealing with complex and large-scale optimization problems by focusing the valuable computational effort to the most promising feasible region while sustaining the global view. It has been proven to converge with probability one to a global optimum (Shi and Olafsson 2000). Since it was developed, it has witnessed many successful applications (Shi and Olafsson 2001; Wu, Shi, el al. 2010). For reviewing the method, the following shows the general procedure of Nested Partition framework.

\section{1) Partitioning}

In each iteration of the NP algorithm, there is a partition of the current most promising region and aggravation of rest part as the surrounding region. The efficiency of an NP algorithm depends on incorporating problem-specific information into the partitioning to impose a useful structure on the search space.

\section{2) Random Sampling}

In each iteration, samples are randomly selected from both of the partitioned promising subregions and the surrounding regions. Different sampling strategies could be applied for different problems and the past experience has shown its great impact on the convergence of the algorithm.

\section{3) Calculation of the Promising Index}

For each subregion and the surrounding region, the promising index function designed is obtained to guide the evaluation of different regions and thus guiding the direction of the next search. 


\section{4) Movement}

With the knowledge of the promising index of different regions, the movement of the current iteration can be made. If the region with best promising index is among the subregions, then it becomes the promising region of the next iteration. Otherwise, the search backtracks to the promising region of the previous iteration.

When implementing NP method it is tricky to find good strategy of partition when the size of the feasible region is large and there is no special structure. To reduce the feasible region and hence improving the efficiency of the method, bounds technique is introduced. By comparing the upper bounds from heuristics and lower bounds, redundant regions that can never generate optimal solution are trimmed and iterative implementation of bounds technique when sampling gives rise to the fast zooming in the promising regions. The details of the solution approach are shown as bellows.

\section{Bounds-based-partitioning}

Instead of partitioning the whole promising region and conducting further examination into each subregion, by conducting bounds-based-partitioning algorithm, the subregion for which it is impossible to contain the optimal point will be trimmed first. And only subregions that are left and can possibly contain optimum will be examined next. The searching region, therefore, is reduced and the efficiency of the method is improved. Specifically, in bounds-based-partitioning algorithm, a good upper bound should be acquired first. In our case, the upper bound can be obtained by selecting the minimal objective value generated by EDD, BJ or FL. For the $k$ th level

node, the lower bound for $L_{\max }$ of the unfathomed partial schedules or $L_{\max }$ of the completed schedules is calculated. According to the result of the lower bound calculation, the region with the lower bound larger than the upper bound would be eliminated. An illustration of the BBP procedure is given in Figure 1.

Figure 1: A illustration on the BBP procedure (Figure 1 will be inserted here)

Bellow shows the procedure of the bounds-based-partitioning algorithm.

Bounds-based-partitioning (BBP) algorithm

Step 1. Implement the algorithm of EDD, BJ and FL and choose the minimal objective value as the upper bound.

Step 2. In the $k$ th level node, the first $k$ positions are occupied by $k$ specific jobs. Let $\Theta$ be the promising region to be partitioned in this level. The initial partitioning procedure would divide $\Theta$ into $n-k$ subregions. 
Step 3. For each subregion acquired above, calculate the lower bound for $L_{\max }$ of the unfathomed partial schedules or $L_{\max }$ of the completed schedules.

Step 4. Eliminate the subregion with the lower bound larger than the upper bound and

obtain the final subregions. Denote $M_{\sigma(k)}$ as the number of subregions obtained in this step. The left subregions to be explored further could be defined as $\sigma_{1}(k), \sigma_{2}(k), \ldots, \sigma_{M_{\sigma(k)}}(k)$.

\section{Bounds-based-sampling}

Sampling is intended to provide the actual reflection of the promising region and the guide on the region studied in the next iteration and even the new upper bound if the objective value obtained is better than the current one. Following the similar logic of bound-based-partitioning algorithm, bounds-based-sampling algorithm introduces bounds to trim the nodes for which it is impossible to generate the optimum and only selects nodes within the remaining promising ones in a random way. Repetition elimination is implemented to avoid repeated sampling through the storage and comparison of the history nodes. The memory for storing the nodes can be emptied once the sampling for one certain subregion is finished. An illustration of the BBS procedure is given in Figure 2.

Figure 2: A illustration on the BBS procedure (Figure 2 will be inserted here)

Bellow shows the procedure of bounds-based-sampling algorithm.

\section{Bounds-based-sampling (BBS) algorithm}

Step 1. As for the current subregion $\sigma_{j}(k)$ for sampling, the $(k+1)$ th level node is prefixed in the partitioning procedure. In the $(k+1)$ th level node, the first $k+1$ positions are occupied by $k+1$ specific jobs. The sampling procedure is conducted through fixing the left level nodes sequentially. For each iteration of the next $n-k-1$ levels, the lower bound is calculated the way elaborated on before.

Step 2. For each followed level, trim the subregions with the lower bound larger than the upper bound.

Step 3. In the $(k+2)$ th level, a random selection is conducted within the remaining subregion by fixing the unfinished job in this position. And the similar procedure is done in the $(k+$ $3) t h,(k+4) t h, \ldots,(n-1) t h$ level.

Step 4. Store the history sample nodes to avoid repeated sampling and refresh it once the sampling of one subregion is finished.

\section{Estimating the promising index and backtracking}

The estimating and backtracking procedure are conducted in the same way as the general Nested Partition framework. The best solution found in a particular region is defined as the 
promising index. In each iteration, the promising index of the explored subregions and that of the surrounding regions are compared. If the surrounding region wins, we would backtrack to the master region of the current promising region and repeat the preceding procedures.

\subsection{BBNP solution framework}

With the proposition of basic procedures of BBNP algorithm, the following shows the details of BBNP-based solution framework that combines the heuristics and branch-and-bound algorithm. And a graphic illustration of the framework will be also given in Figure 3.

\section{BBNP solution framework}

Step 1. Set the time limits BB_TIMELIMIT and BBNP_TIMELIMIT separately for branch-and-bound algorithm and BBNP approach according to the time resources available for problem solution. Let $t_{B B}$ and $t_{B B N P}$ be the counter of time. Initially, $t_{B B}, t_{B B N P}=0$.

Step 2. Implement EDD, BJ and FL algorithm and choose the best solution as the upper bound.

Step 3. Run branch-and-bound algorithm and set $t_{B B}=C U R R E N T T I M E$.

Step 4. If the optimal solution is found or $t_{B B}>B B_{-} T I M E L I M I T$, STOP branch-andbound algorithm. If the optimum is found, STOP the whole solution procedure. Otherwise, go to Step 5 .

Step 5. Set $t_{B B N P}=C U R R E N T T I M E$ and run BBNP approach in the following procedure:

- Step 5-1. Let $d=1$, where $d$ is the counter of the algorithm level; $t=0$, where $t$ is the counter of the time.

- Step 5-2. If $d>n$ or $t>$ TIMELIMIT, STOP.

- Step 5-3. Implement BBP algorithm where $\Theta=$ root region and there are totally $n$ initial subregions and no surrounding region.

- Step 5-4. Apply BBS in each subregion acquired above and the surrounding region $s(\Theta)$.

- Step 5-5. Evaluate the promising index of each subregion and the surrounding region. If the winner is one of the explored subregions, go to Step 5-6. Otherwise, go to Step 5-7.

- Step 5-6. Choose the subregion with the smallest promising index as the next promising region, i.e. $\Theta=\sigma_{j^{*}}(d)$, where $j^{*}$ represents the subregion with smallest promising index. The surrounding region is generated with the other solution space. Set $d=d+1, t=$ CURRENTTIME. 
- Step 5-7. Backtrack to the master region of the current promising region. Set $d=d-1, t=$ CURRENTTIME.

Step 6. If the random searching is completed or $t_{B B N P}>$ BBNP_TIMELIMIT, STOP BBNP approach.

Step \%. Return the current upper bound.

Figure 3: A illustration on the BBNP solution framework (Figure 3 will be inserted here)

\section{Computational experiments}

In this section, computational experiments are conducted to evaluate the effectiveness of the branch-and-bound algorithm and heuristics of EDD, BJ and FL and BBNP. Hypothesis test is implemented to provide a statistical comparison of the performance of different heuristics. In terms of large problem instances for which the optimum can be hardly reached, the performance evaluation of each heuristic is conducted. The algorithms were coded in Visual Studio 2008 and the computational experiments were run on a Pentium 4 with 2 GB RAM personal computer.

The test problems are generated as follows. For each algorithm, the values $a=0.7,0.8,0.9$, and $\alpha=\beta=b=0.5$ are used. For each problem size, 20 random instances are generated. The parameters are generated from uniform distribution in the following parameter: $p_{i j} \in[1,100]$, $d_{j} \in\left(0.0, C_{\overline{\max }}\right] . C_{\overline{\max }}$ can be taken as the makespan acquired by implementing SPT algorithm (where jobs are arranged in the nondecreasing order of $\sum_{i=1}^{M} p_{i j}$ ). For problems of medium size, we consider different pairs of machine number $m$ and job number $n$ by letting $n=9,10,11,12,13,14$ and $m=3,5$. For exploring the performance of heuristics in the case of large size problems, instances are generated by letting $n=20,50,100,150,200$ and $m=5$. The time limit for BBNP approach is set to be $20 \mathrm{~min}$.

In Table 1, computational tests are conducted for medium size problems. As for the branchand-bound algorithm, the average number of nodes, the maximum number of nodes, the average time, and the maximum time (in seconds) were recorded. For the heuristic algorithms, the average and maximum error percentage were recorded. The percentage error of the solution produced by the heuristic algorithm is calculated as

$$
\left(L_{\text {Heur }}-L_{\text {opt }}\right) / L_{\text {opt }} \times 100 \% \text {, }
$$

where $L_{\text {Heur }}$ is the value $L_{\max }$ of the solution by the heuristic algorithms and $L_{\mathrm{opt}}$ is the value $L_{\max }$ of the optimal schedule. As for heuristic algorithms, most problems were solved in no time (the reported CPU time was zero). The running time, therefore, is not given.

Table 1 Computational results of the branch-and-bound, heuristics of EDD, BJ, FL and BBNP approach (Table 1 will be inserted here) 
In Table 2, statistical hypothesis tests are conducted to compare the mean percentage error of each heuristic. The $t$-test is used for the tests: $T=\frac{\overline{X_{\text {Heur } 1}}-\overline{X_{\text {Heur } 2}}}{S_{w} \sqrt{1 / n_{\text {Heur } 1}+1 / n_{\text {Heur } 2}}}$, where $S_{w}^{2}=$ $\frac{\left(n_{\text {Heur } 1}-1\right) S_{\text {Heur } 1}^{2}+\left(n_{\text {Heur } 2}-1\right) S_{\text {Heur } 2}^{2}}{n_{\text {Heur } 1}+n_{\text {Heur } 2}-2}$ and Heur 1, Heur $2 \in\{E D D, B J, F L, B B N P\}$. As the results in Table 1 potentially show that the effectiveness performance of each heuristic is ranked as: $\mathrm{BBNP}>\mathrm{FL}>\mathrm{BJ}>\mathrm{EDD}$, three corresponding statistical hypothesis test are set as $H_{0}: \mu_{E D D}>$ $\mu_{B J}, H_{1}: \mu_{E D D} \leq \mu_{B J} ; H_{0}: \mu_{B J}>\mu_{F L}, H_{1}: \mu_{B J} \leq \mu_{F L} ; H_{0}: \mu_{F L}>\mu_{B B N P}, H_{1}: \mu_{F L} \leq$ $\mu_{B B N P}$. Type $I$ error of $1 \%$ is used and thus $t_{\text {critical }}=2.5$. The results of the hypothesis tests are shown in Table 2.

Table 2 Results of the calculated $t$-values for the hypothesis tests (Table 2 will be inserted here)

In Table 3, large-sized problems are generated for further evaluation of the performance of heuristics. The performance of each heuristic is verified using the average and maximum ratio $\frac{L_{\max }(H e u r)}{L_{\max }(E D D)}$, where Heur $\in\{B J, F L, B B N P\}$.

Table 3 Computational results of the heuristics for large-scale data size (Table 3 will be inserted here)

\section{Summary}

In this paper, we studied flow shop scheduling with a general exponential time-dependent learning effect. As far as we concern, the problem with the objective of minimizing the maximum of lateness is first discussed in this model. For better understanding of the problem, an equivalent problem and a special instance are given. To provide a hybrid solution framework, two heuristics, a branch-and-bound algorithm and an NP-based approach are proposed. Some dominance rules and composite bounds are developed to provide mathematical guide on the solution framework and improve the efficiency of the approach.

\section{References}

[1] Allahverdi, A., Aldowaisan, T. (2004). No-wait flowshops with bicriteria of makespan and maximum lateness. European Journal of Operational Research, 152, 132-147.

[2] Bai, J., Wang, M-Z, Wang, J-B (2012). Single machine scheduling with a general exponential learning effect. Applied Mathematical Modelling, 36, 829-835.

[3] Bachman, A., Janiak, A. (2000). Minimizing maximum lateness under linear deterioration. European Journal of Operational Research, 126, 557-566.

[4] Biskup, D. (1999). Single-machine scheduling with learning considerations. European Journal of Operational Research, 115, 173-178. 
[5] Biskup, D. (2008). A state-of-the-art review on scheduling with learning effects. European Journal of Operational Research, 188, 315-329.

[6] Cheng, T.C.E., Wang, G. (2000). Single machine scheduling with learning effect considerations. Annals of Operations Research, 98, 273-290.

[7] Cheng, T.C.E., Ding, Q., Lin, B.M.T. (2004). A concise survey of scheduling with timedependent processing times. European Journal of Operational Research, 152, 1-13.

[8] Cheng, T.C.E., Wu, C.C., Lee, W.C. (2008). Some scheduling problems with sum-ofprocessing-times-based and job-position-based learning effects, Information Sciences, 178, $2476 \mathrm{C} 2487$.

[9] Cheng, T.C.E., Kuo, W-H, Yang, D-L (2013). Scheduling with a position-weighted learning effect based on sum-of-logarithm-processing-times and job position. Information Sciences, $221,490 \mathrm{C} 500$.

[10] Day, G.S., Montgomery, D.B. (1983). Diagnosing the experience curve. Journal of Marketing, 47, 44-58.

[11] Framinan, J.M., Leisten, R. (2003). An efficient constructive heuristic for flowtime minimization in permutation flow shops. OMEGA: The International Journal of Management Science, 31, 311-317.

[12] Gonzalez, T., Sahni, S. (1978). Flowshop and jobshop schedules: Complexity and approximation. Operations Research, 26, 36-52.

[13] Garey, M.R., Johnson, D.S. (1979). Computers and Intractability: A Guided Tour to The Theory of NP-Completeness Freeman: San Francisco.

[14] Haouari, M., Ladhari, T. (2000). Minimising maximum lateness in a two-machine flowshop. Journal of the Operational Research Society, 51, 1100-1106.

[15] Hsu, Y.S., Lin, B.M.T. (2003). Minimization of maximum lateness under linear deterioration. Omega, 31, 459-469.

[16] Johnson, S.M. (1954). Optimal two-an-tree-stage production schedules with setup times included. Naval Research Logistics, 1, 61-68.

[17] Janiak, A., Rudek, R (2009). Experience based approach to scheduling problems with the learning effect. IEEE Transactions on Systems, Man and Cybernetics Part A, 2, 344-57.

[18] Koulamas, C., Kyparisis, G.J. (2007). Single-machine and two-machine flowshop scheduling with general learning functions. European Journal of Operational Research, 178, 402-407. 
[19] Kuo, W-H, Hsu, C-J, Yang, D-L (2012). Worst-case and numerical analysis of heuristic algorithms for flow shop scheduling problems with a time-dependent learning effect. Journal of Information Sciences, 184, 282C97.

[20] Kuo, W-H, Yang, D-L (2006). Minimizing the makespan in a single machine scheduling problem with a time-based learning effect. Information Processing Letters, 97, 64-67.

[21] Lee, W-C, Wu, C-C (2009). Some single-machine and m-machine flowshop scheduling problems with learning considerations. Journal of Information Sciences, 179, 3885-92.

[22] Pinedo, M. (2002). Scheduling: Theory, Algorithms and Systems Prentice hall, Englewood cliffs, New Jersey.

[23] Smutnicki, C. (1998). Some results of the worst-case analysis for flow shop scheduling. European Journal of Operational Research, 109, 66-87.

[24] Shi, L., Olafsson, S. (2000). Nested Partitions method for global optimization. Operations Research, 26, 371-394.

[25] Shi, L., Olafsson, S. (2001). An optimization framework for product design. Management Science, 47, 1681-1692.

[26] Venezia, I. (1985). On the statistical origins of the learning curve. European Journal of Operational Research, 19, 191-200.

[27] Xu, Z., Sun, L., Gong, J. (2008). Worst-case analysis for flow shop scheduling with a learning effect. International Journal of Production Economics, 113, 748-753.

[28] Wu, T., Shi, L., Neil, A.D. (2010). An HNP-MP approach for the capacitated multi-Item lot sizing problem with setup times. IEEE Transactions on Automation Science and Engineering, $7,500-511$.

[29] Webb, G.K. (1994). Integrated circuit (IC) pricing. High Technology Management Research, 5, 247-260.

[30] Wang, J-B., Xia, Z-Q (2005). Flow shop scheduling with a learning effect. Journal of Operational Research Society, 56, 1325-30.

[31] Wang, J-B, Wang, D., Wang, L-Y, Lin, L., Yin, N., Wang, W-W (2009). Single machine scheduling with exponential time-dependent learning effect and past-sequence-dependent setup times. Computers and Mathematics with Applications, 57(1), 9-16.

[32] Wang, J-B, Wang, M-Z (2011). Worst-case behavior of simple sequencing rules in flow shop scheduling with general position-dependent learning effects. Annals of Operations Research, 191, 155-169. 
[33] Wang, J-B, Wang, M-Z (2012). Worst-case analysis for flow shop scheduling problems with an exponential learning effect. Journal of the Operational Research Society, 63, 130-137.

[34] Wang, X-Y, Shou, Z., Zhang, X., Ji, P., Wang, J-B (2013). Several flow shop scheduling problems with truncated position-based learning effect. Computers and Operations Research, 40, 2906-29.

[35] Wang, J-B, Wang, J-J (2014). Flowshop scheduling with a general exponential learning effect. Computers and Operations Research, 43, 292-308.

[36] Wright, T.P. (1936). Factors affecting the cost of airplanes. Journal of Aeronautical Sciences, 3, 122-128. 
Table 1 Results of the branch-and-bound, heuristics of EDD, BJ, FL and BBNP approach

\begin{tabular}{|c|c|c|c|c|c|c|c|c|c|c|c|c|c|c|}
\hline \multirow[b]{3}{*}{$m$} & \multirow[b]{3}{*}{$n$} & \multicolumn{5}{|c|}{ branch and bound algorithms } & \multicolumn{4}{|c|}{ error percentage (\%) } & \multirow{2}{*}{\multicolumn{2}{|c|}{ FL }} & \multirow{2}{*}{\multicolumn{2}{|c|}{ BBNP }} \\
\hline & & & \multicolumn{2}{|c|}{ CPU time $(\mathrm{s})$} & \multicolumn{2}{|c|}{ node number } & \multicolumn{2}{|c|}{ EDD } & \multicolumn{2}{|c|}{$\mathrm{BJ}$} & & & & \\
\hline & & $a$ & mean & $\max$ & mean & $\max$ & mean & $\max$ & mean & $\max$ & mean & $\max$ & mean & $\max$ \\
\hline \multirow{3}{*}{3} & \multirow{3}{*}{9} & 0.7 & 0.0 & 0.4 & 899 & 8165 & 12.8 & 40.1 & 2.19 & 11.3 & 0.035 & 0.579 & 0.0 & 0.0 \\
\hline & & 0.8 & 0.0 & 0.3 & 653 & 4980 & 12.1 & 30.0 & 2.98 & 15.9 & 0.065 & 0.687 & 0.0 & 0.0 \\
\hline & & 0.9 & 0.0 & 0.3 & 556 & 5168 & 12.3 & 35.3 & 2.59 & 14.2 & 0.069 & 0.778 & 0.0 & 0.135 \\
\hline \multirow{3}{*}{3} & \multirow{3}{*}{10} & 0.7 & 0.0 & 0.3 & 600 & 7110 & 14.0 & 29.9 & 2.91 & 15.8 & 0.171 & 2.91 & 0.0 & 0.0 \\
\hline & & 0.8 & 0.3 & 3.5 & 4689 & 51369 & 14.3 & 35.3 & 3.01 & 17.6 & 0.112 & 2.13 & 0.0 & 0.11 \\
\hline & & 0.9 & 0.2 & 1.1 & 2369 & 32561 & 14.9 & 37.5 & 2.15 & 10.3 & 0.151 & 1.91 & 0.0 & 0.0 \\
\hline \multirow{3}{*}{3} & \multirow{3}{*}{11} & 0.7 & 2.9 & 15.9 & 39112 & 220031 & 15.1 & 26.9 & 2.86 & 10.1 & 0.406 & 3.56 & 0.081 & 1.23 \\
\hline & & 0.8 & 1.4 & 22.1 & 14986 & 289632 & 14.1 & 29.6 & 2.35 & 9.96 & 0.298 & 2.556 & 0.043 & 1.56 \\
\hline & & 0.9 & 2.7 & 35.9 & 40013 & 670101 & 13.1 & 35.6 & 1.99 & 7.68 & 0.420 & 4.33 & 0.0 & 0.0 \\
\hline \multirow{3}{*}{3} & \multirow{3}{*}{12} & 0.7 & 0.3 & 0.6 & 51 & 419 & 14.6 & 35.6 & 1.85 & 6.16 & 0.164 & 2.36 & 0.1 & 0.015 \\
\hline & & 0.8 & 4.5 & 85.1 & 54568 & 1005689 & 15.9 & 38.6 & 1.84 & 5.48 & 0.364 & 3.21 & 0.071 & 0.988 \\
\hline & & 0.9 & 0.2 & 2.6 & 2132 & 231659 & 14.2 & 35.9 & 2.46 & 9.13 & 0.371 & 4.06 & 0.122 & 1.77 \\
\hline \multirow{3}{*}{3} & \multirow{3}{*}{13} & 0.7 & 22.1 & 498.3 & 287869 & 5291659 & 16.8 & 32.6 & 2.39 & 9.80 & 0.368 & 4.11 & 0.166 & 1.87 \\
\hline & & 0.8 & 23.5 & 378.1 & 281654 & 4959654 & 17.1 & 32.1 & 2.65 & 7.56 & 0.371 & 2.39 & 0.101 & 0.936 \\
\hline & & 0.9 & 5.9 & 112.3 & 61123 & 1213659 & 14.9 & 25.1 & 2.81 & 6.73 & 0.263 & 2.12 & 0.029 & 0.301 \\
\hline & & 0.7 & 612.1 & 11235.1 & 7743298 & 153121698 & 12.1 & 22.4 & 1.71 & 7.32 & 0.109 & 1.12 & 0.049 & 0.890 \\
\hline 3 & 14 & 0.8 & 296.1 & 3598.1 & 3471698 & 378936598 & 13.1 & 20.9 & 2.19 & 8.68 & 0.303 & 2.39 & 0.131 & 1.75 \\
\hline & & 0.9 & 121.3 & 1975.3 & 1349965 & 20896987 & 13.9 & 24.9 & 2.45 & 8.19 & 0.212 & 2.39 & 0.098 & 1.203 \\
\hline & & 0.7 & 0.1 & 0.3 & 469 & 3713 & 16.1 & 42.9 & 2.91 & 11.5 & 0.528 & 3.46 & 0.316 & 3.41 \\
\hline 5 & 9 & 0.8 & 0.1 & 0.5 & 623 & 5415 & 13.5 & 28.9 & 4.19 & 12.9 & 0.798 & 4.81 & 0.311 & 1.85 \\
\hline & & 0.9 & 0.1 & 0.5 & 359 & 2986 & 15.1 & 35.5 & 3.61 & 11.2 & 0.799 & 3.09 & 0.275 & 2.16 \\
\hline & & 0.7 & 0.2 & 2.1 & 1781 & 9631 & 17.9 & 36.59 & 3.33 & 16.9 & 0.785 & 3.28 & 0.495 & 3.25 \\
\hline 5 & 10 & 0.8 & 0.2 & 2.9 & 2001 & 20235 & 18.1 & 35.3 & 3.31 & 8.12 & 0.992 & 5.19 & 0.401 & 3.56 \\
\hline & & 0.9 & 0.1 & 1.1 & 771 & 4611 & 16.1 & 26.9 & 3.11 & 10.8 & 0.565 & 3.53 & 0.323 & 2.99 \\
\hline & & 0.7 & 3.3 & 24.0 & 19305 & 144002 & 18.5 & 38.4 & 4.19 & 19.4 & 0.748 & 3.12 & 0.412 & 2.16 \\
\hline 5 & 11 & 0.8 & 0.8 & 3.3 & 2068 & 10265 & 16.9 & 30.0 & 2.29 & 9.15 & 0.988 & 4.66 & 0.658 & 4.58 \\
\hline & & 0.9 & 2.2 & 22.9 & 12005 & 135689 & 19.1 & 41.5 & 2.77 & 8.18 & 0.648 & 3.68 & 0.216 & 1.26 \\
\hline & & 0.7 & 7.3 & 105.6 & 60023 & 939865 & 18.8 & 28.9 & 3.41 & 12.0 & 1.26 & 5.66 & 1.11 & 5.28 \\
\hline 5 & 12 & 0.8 & 3.5 & 42.9 & 23568 & 297986 & 20.6 & 36.9 & 5.69 & 12.1 & 1.21 & 6.31 & 0.558 & 2.54 \\
\hline & & 0.9 & 33.1 & 431.9 & 209986 & 2916895 & 15.5 & 30.9 & 3.58 & 11.4 & 0.559 & 2.31 & 0.356 & 1.86 \\
\hline & & 0.7 & 43.9 & 481.6 & 206598 & 2279865 & 20.1 & 43.9 & 3.49 & 14.0 & 1.21 & 3.66 & 0.544 & 2.98 \\
\hline 5 & 13 & 0.8 & 85.5 & 1035.2 & 519865 & 6269865 & 18.8 & 39.5 & 2.91 & 8.75 & 0.181 & 1.77 & 0.021 & 0.475 \\
\hline & & 0.9 & 26.2 & 388.1 & 156895 & 2391000 & 15.9 & 33.6 & 2.20 & 9.92 & 0.579 & 4.33 & 0.279 & 2.11 \\
\hline & & 0.7 & 2112.1 & 29632.1 & 13351654 & 196658659 & 20.3 & 33.1 & 2.99 & 9.24 & 0.986 & 4.88 & 0.495 & 2.01 \\
\hline 5 & 14 & 0.8 & 111.3 & 889.8 & 554659 & 5557956 & 20.1 & 37.1 & 3.26 & 9.13 & 1.22 & 8.56 & 0.886 & 4.21 \\
\hline & & 0.9 & 236.5 & 4728.1 & 1404659 & 28115649 & 16.9 & 32.3 & 2.86 & 12.9 & 0.586 & 2.69 & 0.365 & 2.31 \\
\hline
\end{tabular}


Table(s)

Table 2 Calculated $t$-values for the hypothesis tests

\begin{tabular}{|c|c|c|c|c|c|}
\hline$m$ & $n$ & $a$ & $T_{1}$ & $T_{2}$ & $T_{3}$ \\
\hline \multirow{3}{*}{3} & \multirow{3}{*}{9} & 0.7 & 4.6968 & 4.0865 & 3.3568 \\
\hline & & 0.8 & 4.0321 & 4.1549 & 3.3687 \\
\hline & & 0.9 & 4.3265 & 4.0165 & 3.3325 \\
\hline \multirow{3}{*}{3} & \multirow{3}{*}{10} & 0.7 & 4.1358 & 3.9658 & 4.2568 \\
\hline & & 0.8 & 4.1568 & 3.9986 & 4.0123 \\
\hline & & 0.9 & 4.1986 & 4.0568 & 3.9658 \\
\hline \multirow{3}{*}{3} & \multirow{3}{*}{11} & 0.7 & 4.2156 & 3.8659 & 3.2658 \\
\hline & & 0.8 & 4.5638 & 3.7658 & 3.0568 \\
\hline & & 0.9 & 4.5986 & 3.5986 & 3.9865 \\
\hline \multirow{3}{*}{3} & \multirow{3}{*}{12} & 0.7 & 4.5998 & 3.8562 & 3.9685 \\
\hline & & 0.8 & 4.6123 & 3.6581 & 4.1352 \\
\hline & & 0.9 & 4.6012 & 3.7685 & 4.1589 \\
\hline \multirow{3}{*}{3} & \multirow{3}{*}{13} & 0.7 & 4.4231 & 3.7745 & 4.1256 \\
\hline & & 0.8 & 4.3123 & 3.8965 & 3.9865 \\
\hline & & 0.9 & 4.0216 & 3.6986 & 3.9876 \\
\hline \multirow{3}{*}{3} & \multirow{3}{*}{14} & 0.7 & 3.9865 & 3.6875 & 2.5189 \\
\hline & & 0.8 & 3.8968 & 3.7564 & 3.1356 \\
\hline & & 0.9 & 4.1564 & 3.6998 & 3.0658 \\
\hline \multirow{3}{*}{5} & \multirow{3}{*}{9} & 0.7 & 4.6785 & 3.8965 & 1.2385 \\
\hline & & 0.8 & 4.1568 & 3.9685 & 3.9945 \\
\hline & & 0.9 & 4.3596 & 4.0124 & 3.1358 \\
\hline \multirow{3}{*}{5} & \multirow{3}{*}{10} & 0.7 & 4.2139 & 4.1763 & 2.5014 \\
\hline & & 0.8 & 4.4318 & 2.6885 & 3.0156 \\
\hline & & 0.9 & 3.8745 & 3.8795 & 2.9865 \\
\hline \multirow{3}{*}{5} & \multirow{3}{*}{11} & 0.7 & 3.6986 & 4.2158 & 2.8568 \\
\hline & & 0.8 & 4.3128 & 3.7587 & 2.1587 \\
\hline & & 0.9 & 4.5895 & 3.7785 & 3.3239 \\
\hline \multirow{3}{*}{5} & \multirow{3}{*}{12} & 0.7 & 3.4328 & 3.8846 & 2.5516 \\
\hline & & 0.8 & 3.7745 & 3.5754 & 3.9948 \\
\hline & & 0.9 & 3.5876 & 4.0358 & 2.9985 \\
\hline \multirow{3}{*}{5} & \multirow{3}{*}{13} & 0.7 & 4.4118 & 4.0998 & 2.8765 \\
\hline & & 0.8 & 4.5116 & 4.1008 & 3.9984 \\
\hline & & 0.9 & 4.1895 & 3.5489 & 3.5411 \\
\hline \multirow{3}{*}{5} & \multirow{3}{*}{14} & 0.7 & 4.2587 & 3.4185 & 2.9759 \\
\hline & & 0.8 & 4.4965 & 1.9851 & 3.8795 \\
\hline & & 0.9 & 4.0156 & 4.2185 & 2.0584 \\
\hline
\end{tabular}


Table(s)

Table 3 Computational results of the heuristics for large-scale data size

\begin{tabular}{|c|c|c|c|c|c|c|c|c|}
\hline \multirow[b]{2}{*}{$m$} & \multirow[b]{2}{*}{$n$} & \multirow[b]{2}{*}{$a$} & \multicolumn{2}{|c|}{$\frac{L_{\max }(B J)}{L_{\max }(E D D)}$} & \multicolumn{2}{|c|}{$\frac{L_{\max }(F L)}{L_{\max }(E D D)}$} & \multicolumn{2}{|c|}{$\frac{L_{\max }(B B N P)}{L_{\max }(E D D)}$} \\
\hline & & & mean & $\max$ & mean & $\max$ & mean & $\max$ \\
\hline \multirow{3}{*}{5} & & 0.7 & 0.8375 & 0.9709 & 0.7385 & 0.8903 & 0.7291 & 0.8671 \\
\hline & 20 & 0.8 & 0.8201 & 0.9444 & 0.7158 & 0.8564 & 0.7018 & 0.8176 \\
\hline & & 0.9 & 0.8269 & 0.9545 & 0.7169 & 0.8625 & 0.7054 & 0.8501 \\
\hline \multirow{3}{*}{5} & & 0.7 & 0.8378 & 0.9594 & 0.7168 & 0.8601 & 0.6905 & 0.8113 \\
\hline & 40 & 0.8 & 0.8026 & 0.9553 & 0.7405 & 0.8828 & 0.6886 & 0.8316 \\
\hline & & 0.9 & 0.8132 & 0.9424 & 0.7041 & 0.8601 & 0.6951 & 0.8525 \\
\hline \multirow{3}{*}{5} & & 0.7 & 0.8307 & 0.9597 & 0.7168 & 0.8694 & 0.7023 & 0.8416 \\
\hline & 60 & 0.8 & 0.8258 & 0.9645 & 0.7162 & 0.8702 & 0.6886 & 0.8328 \\
\hline & & 0.9 & 0.8234 & 0.9308 & 0.7039 & 0.8380 & 0.6880 & 0.7857 \\
\hline \multirow{3}{*}{5} & & 0.7 & 0.8339 & 0.9783 & 0.7280 & 0.8896 & 0.6699 & 0.8026 \\
\hline & 80 & 0.8 & 0.8150 & 0.9513 & 0.6924 & 0.8327 & 0.6707 & 0.8483 \\
\hline & & 0.9 & 0.7982 & 0.9187 & 0.6863 & 0.8526 & 0.6658 & 0.8208 \\
\hline \multirow{3}{*}{5} & & 0.7 & 0.7302 & 0.8983 & 0.6930 & 0.8742 & 0.6340 & 0.8256 \\
\hline & 100 & 0.8 & 0.7984 & 0.9147 & 0.6806 & 0.8219 & 0.6593 & 0.7919 \\
\hline & & 0.9 & 0.7547 & 0.9115 & 0.6282 & 0.8448 & 0.6135 & 0.8138 \\
\hline
\end{tabular}


Figure(s)

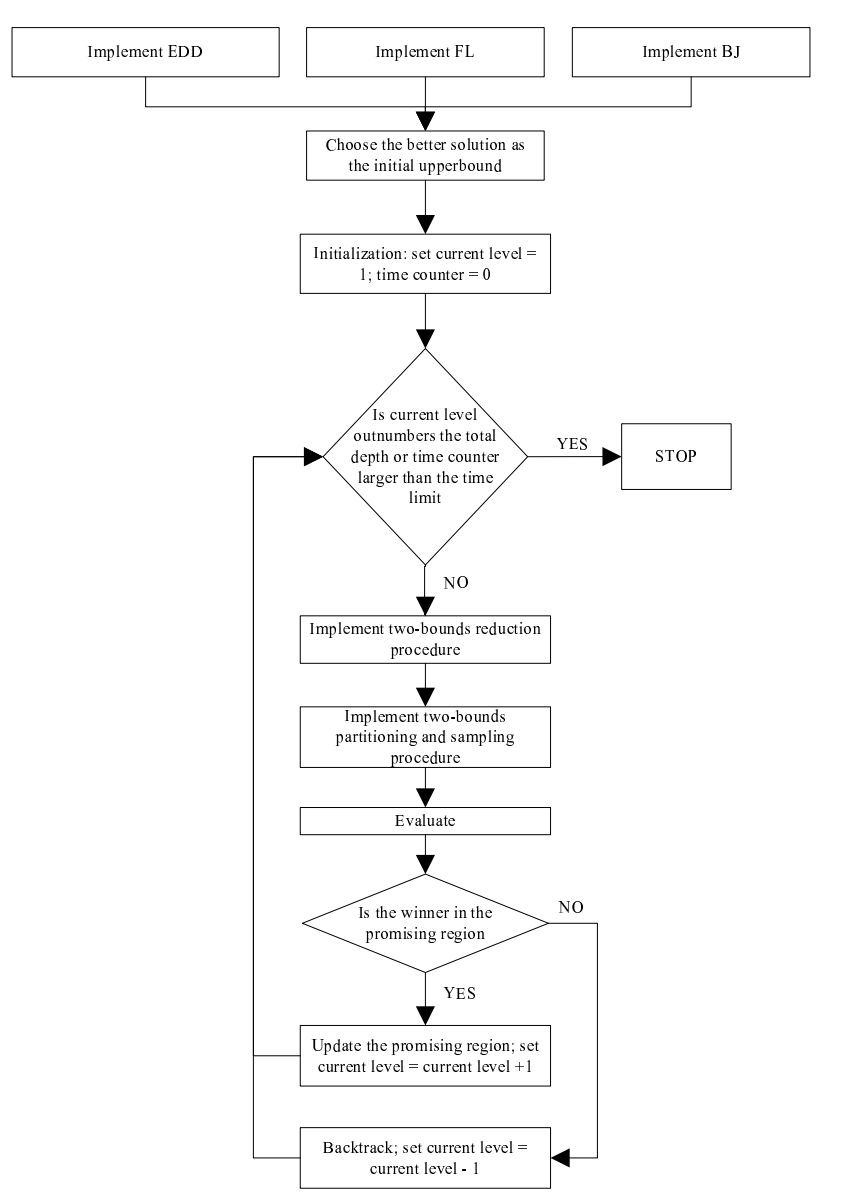

Figure 3: A illustration on the BBNP solution framework

.




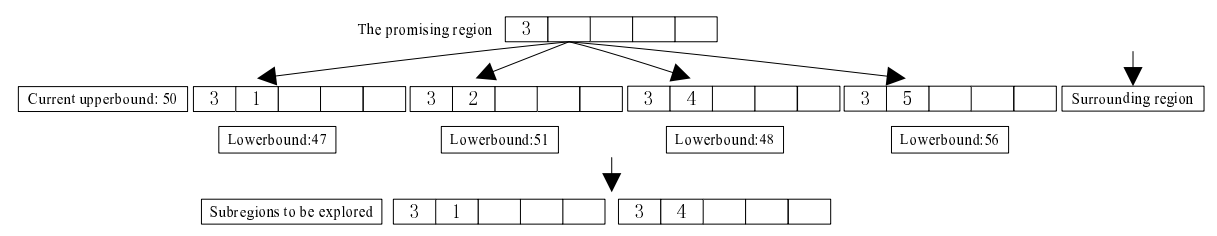

Figure 1: A illustration on the BBP procedure

Figure(s) 
Figure(s)

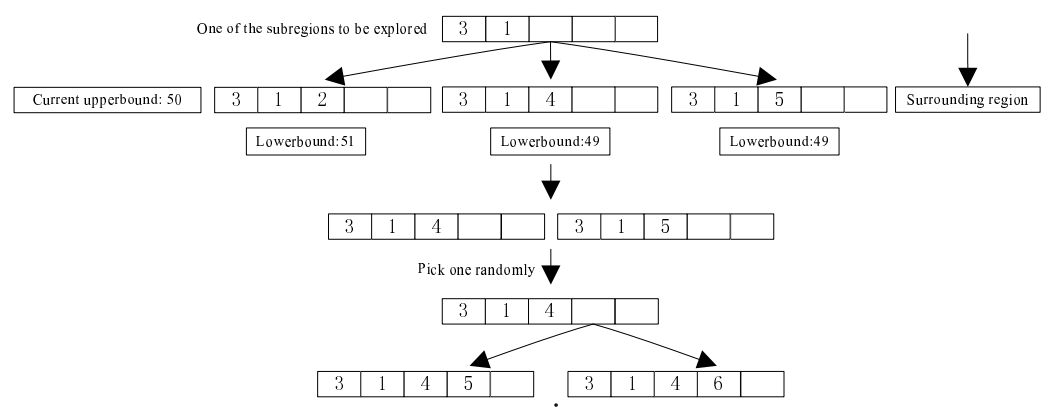

Figure 2: A illustration on the $\mathrm{BBP}$ procedure 
Acknowledgement

\section{Acknowledgements}

This paper was supported by National Science Foundation of China(NSFC) under Grant 71371015 and National science and technology support plan special funds of China under grant No.2013BAD17B08. 